\title{
Composição corporal em mulheres com deficiência da 21-hidroxilase: comparação dos métodos antropométricos e de impedância bioelétrica em relação a um grupo controle
}

\author{
Body composition in females with 21-hydroxylase \\ deficiency: comparison of anthropometric methods and \\ bioelectric impedance in relation to a control group
}

Ezequiel Moreira Gonçalves ${ }^{1,2,3}$, Sofia Helena Valente de Lemos-Marini ${ }^{4}$, Maricilda Palandi de Mello, Alexandre Duarte Baldin', Wellington Roberto Gomes Carvalho ${ }^{1,2}$, Edson Santos Farias ${ }^{1,6}$, Gil Guerra-Júnior ${ }^{1,4}$

\footnotetext{
'Laboratório de Crescimento e Composição Corporal, Centro de Investigação em Pediatria (Ciped), Faculdade de Ciências Médicas (FCM), Universidade Estadual de Campinas (Unicamp), Campinas, SP, Brasil

2 Programa de Pós-Graduação em Saúde da Criança e do Adolescente FCM-Unicamp, Campinas, SP, Brasil ${ }^{3}$ Grupo de Estudo e Pesquisa em Nutrição, Metabolismo e Exercício, Universidade Estadual de Londrina (UEL), Londrina, PR, Brasil

${ }^{4}$ Departamento de Pediatria, FCM-Unicamp, Campinas, SP, Brasil ${ }^{5}$ Centro de Biologia Molecular e Engenharia Genética (CBMEG), Unicamp, Campinas, SP, Brasil ${ }^{6}$ Departamento de Educação Física e Desporto, Universidade Federal do Acre (UFAC), Rio Branco, AC, Brasil
}

Correspondência para: Gil Guerra-Júnior

Departamento de Pediatria, FCMUnicamp

Caixa Postal 6111

13083-970 - Campinas, SP, Brasil gilguer@fcm.unicamp.br

Recebido em 11/Maio/2009 Aceito em 2/Nov/2009

\section{RESUMO}

Objetivo: Estimar a composição corporal avaliada pela espessura de dobras cutâneas (EDC) e pela impedância bioelétrica (BIA) em um grupo de mulheres com hiperplasia adrenal congênita, forma clássica por deficiência da enzima 21-hidroxilase (HAC-C-D21OH) e em relação ao um grupo controle. Pacientes e métodos: Foram avaliadas 16 pacientes com HAC-C-D210H e 26 controles, todas do sexo feminino, com idades de 8 a 18 anos. Foram estimadas as massas gorda (MG) e magra (MM) utilizando EDC e BIA. Os dados das MG e MM foram analisados de forma relativa (\%), absoluta $(\mathrm{kg})$ e em relação à estatura (IMG e IMM). Resultados: Não foram encontradas diferenças significativas entre os dois métodos em cada grupo avaliado. Em relação ao grupo controle, as pacientes com HAC-C-D21OH apresentaram valores maiores de MG (\%) BIA, IMG BIA e IMM EDC e menores de MM (\%) BIA. Foi verificada correlação positiva e alta em todos os parâmetros analisados. Conclusão: Os resultados observados pelos métodos de EDC e BIA foram semelhantes nesta amostra de mulheres com HAC-C$\mathrm{D} 210 \mathrm{H}$, porém controversos em relação ao grupo controle, sugerindo que se tenha cautela no uso desses métodos de avaliação da composição corporal nessa população. Arq Bras Endocrinol Metab. 2010;54(3):274-81

\section{Descritores}

Hiperplasia suprarrenal congênita; antropometria; impedância bioelétrica; dobras cutâneas; adiposidade

\section{ABSTRACT}

Objective: To estimate body composition by evaluating skinfold thickness (ST) and electric bioimpedance $(\mathrm{EB})$ in a group of women with congenital adrenal hyperplasia due to classical 21-hydroxylase deficiency (CAH-C-210HD) in relation to a control group. Subjects and methods: Sixteen patients and 26 controls were evaluated, all female, with age varying from 8 to 18 years. Fat (FM) and lean (LM) masses were evaluated by ST and EB. FM and LM data were analyzed in relative (\%) and absolute $(\mathrm{kg})$ values, and in relation to stature (FMI and LMI). Results: There were no observed significant differences between the two methods in each group. In relation to the control group, CAH-C-210HD patients showed higher values for FM (\%) EB, FMI EB and LMI ST but lower values for FM (\%) EB. Positive and high correlation values were observed for all parameters analyzed. Conclusion: ST and EB results observed were similar in this sample of females with CAH-C-21OHD, but controversial in relation to the control group, suggesting caution when using these methods to evaluate body composition in this population. Arq Bras Endocrinol Metab. 2010;54(3):274-81

\section{Keywords}

Congenital adrenal hyperplasia; anthropometry; electric impedance; skinfold thickness; adiposity 


\section{INTRODUÇÃO}

A forma clássica da hiperplasia adrenal congênita (HAC-C) é caracterizada pela deficiência em uma das enzimas que participam do metabolismo do cortisol, e sua forma mais comum ocorre por deficiência da 21-hidroxilase (HAC-C-D21OH), responsável por cerca de $95 \%$ dos casos, apresentando as variantes virilizante simples (VS), com produção deficiente de cortisol e aumentada de andrógenos, e perdedora de sal (PS), que, além dos sintomas observados na variante VS, apresenta comprometimento da produção de mineralocorticoides (1-3).

Tão logo é feito o diagnóstico, é indispensável o uso de glicocorticoide associado ou não a mineralocorticoide de forma substitutiva para o controle da doença. O tratamento de reposição com glicocorticoide, apesar dos avanços, ainda é de difícil controle e pode levar tanto à situação de tratamento em subdose, com sinais de hiperandrogenismo, como virilização em ambos os gêneros, aumento da massa magra, aceleração da maturação esquelética, fusão prematura das cartilagens de crescimento e baixa estatura final, quanto à situação de tratamento com dose excessiva, com sinais de hipercortisolismo, acarretando déficit de crescimento, aumento da gordura corporal e osteoporose $(4,5)$. Além disso, existem evidências claras de que os pacientes com HAC-C-D21OH, em terapia de substituição hormonal, apresentam risco aumentado de obesidade e complicações metabólicas relacionadas ao tratamento (6).

A impedância bioelétrica (BIA) e a medida antropométrica de espessura das dobras cutâneas (EDC) são técnicas de campo, não invasivas, utilizadas amplamente por profissionais e pesquisadores da área da saúde, na análise da composição corporal, sobretudo, pela sua facilidade de aplicação e de transporte, baixo custo operacional, possibilidade de avaliar um grande número de pessoas num pequeno período de tempo e possuir um grau de simplicidade e confiabilidade relativamente alto, desde que as recomendações inerentes aos seus protocolos sejam rigidamente seguidas, apesar de serem métodos duplamente indiretos. A BIA baseia-se no princípio de que a resistência a uma determinada corrente elétrica é inversamente proporcional à distribuição de água corporal total e de eletrólitos (7). Já a EDC baseia-se no princípio de que há uma relação entre as somatórias de dobras cutâneas e a densidade corporal (8).

A utilização da BIA e da EDC na estimativa da composição corporal pode facilitar o acesso a impor- tantes informações para o monitoramento da HAC-CD21OH. Entretanto, esses métodos ainda necessitam de estudos nesse tipo de população, visto que, em sua origem, foram desenvolvidos para sujeitos saudáveis.

Portanto, o objetivo deste estudo foi avaliar a composição corporal estimada por dois métodos não invasivos (EDC e BIA) em um grupo de mulheres com HAC-C-D21OH e em relação ao um grupo de mulheres sem a doença.

\section{SUJEITOS E MÉTODOS}

Trata-se de um estudo transversal, no qual o grupo denominado "casos" foi constituído por 16 mulheres com idades de oito a 18 anos e diagnóstico clínico, hormonal e molecular de HAC-C-D21OH (9-12). Desse total, 12 apresentavam a variante clínica PS e quatro, VS. Todas iniciaram o acompanhamento no Ambulatório de Endocrinologia Pediátrica do Hospital de Clínicas (HC) da Universidade Estadual de Campinas (Unicamp), nos primeiros meses de vida (tempo médio de tratamento $12,5 \pm 3,0$ anos). Dessas 16 pacientes, 10 foram classificadas como tendo bom controle (BC) da doença e seis, com mau controle (MC), levando-se em consideração o controle hormonal e a aderência ao tratamento. Foram classificadas como bem controladas as pacientes que, além da aderência ao tratamento, apresentaram $50 \%$ ou mais das dosagens séricas de androstenediona e $17 \mathrm{OH}$-progesterona dentro dos valores normais para gênero, idade e desenvolvimento puberal. Foram avaliadas todas as dosagens séricas encontradas no prontuário de cada paciente e que são realizadas, em geral, com intervalo de três meses. Em todas as pacientes do grupo casos, foi realizada a avaliação de maturação esquelética (idade óssea) pelo método TW2 20 ossos (13), avaliada por um dos autores (GGJr).

No grupo denominado "controles", foram incluídas 26 mulheres saudáveis, pareadas por idade em relação à idade óssea das pacientes do grupo casos. As participantes desse grupo estavam matriculadas em uma escola da rede pública estadual do interior do Estado de São Paulo.

O estudo foi aprovado pelo Comitê de Ética em Pesquisa da Faculdade de Ciências Médicas (FCM) da Unicamp (Parecer 219/2005), e todos os sujeitos foram incluídos no estudo após a assinatura do termo de consentimento pós-informado por parte dos responsáveis.

Foram utilizadas medidas antropométricas para a determinação da estatura $(\mathrm{cm})$, utilizando um antropômetro vertical com precisão de $0,1 \mathrm{~cm}$, do peso corpo- 
ral ( $\mathrm{kg}$ ) avaliado em balança eletrônica, calibrada, com precisão de $100 \mathrm{~g}$, dos perímetros da cintura $(\mathrm{cm})$ e do quadril $(\mathrm{cm})$, utilizando-se uma fita metálica com escala de precisão de $0,1 \mathrm{~cm}$, de acordo com as técnicas descritas por Lohman e cols. (14). A partir dessas medidas, foram calculados o índice de massa corporal (IMC = $\mathrm{kg} / \mathrm{m}^{2}$ ) e a relação cintura-quadril (RCQ), obtida pela divisão do perímetro da cintura pelo do quadril.

Os valores absolutos de peso, estatura e IMC foram transformados em escores de desvio-padrão $(z)$, utilizando-se como referência os dados do National Center for Health Statistics (NCHS) de 2000 (15).

Para a avaliação da composição corporal, foram utilizados os métodos de BIA e EDC. A BIA foi realizada utilizando um aparelho tetrapolar BIA 101 Q (RJL Systems, Detroit, EUA), que gera uma corrente de $800 \mu \mathrm{A}$ com frequência de $50 \mathrm{kHz}$. Em ambiente com temperatura e umidade controladas, os sujeitos foram colocados em decúbito dorsal em uma superfície não condutora, com os braços e pernas abduzidos em ângulo de aproximadamente $45^{\circ}$, para que não houvesse o contato entre os braços, o tronco e as coxas. Após a limpeza da pele com álcool, foram colocados quatro eletrodos: dois na mão e dois no pé, ambos do lado direito de acordo com as especificações descritas por Sardinha e cols. (16). Assim como o protocolo recomendado por Heyward e Stolarczyk (8), todos os sujeitos foram orientados a: a) não comer ou beber quatro horas antes do exame; $b$ ) não fazer exercícios 12 horas antes do exame; c) urinar 30 minutos antes do exame; d) não consumir álcool 48 horas antes do exame; e) não fazer uso de diuréticos sete dias antes do exame, f) não estar no período menstrual, e g) não utilizar objetos de metal (brincos, relógios, pulseiras etc.) durante o exame.

Após essas medidas, foram calculados os valores relativos (\%MG BIA e \%MM BIA) e absolutos (MG BIA e MM BIA em kg) dos componentes da composição corporal, utilizando o programa Weight Manager 2.05c, fornecido pelo fabricante (RJL Systems, Detroit, EUA).

Também foram medidas as EDC nas regiões subescapular $(\mathrm{Se})$ e triciptal (Tri), por um único avaliador experiente e previamente treinado (EMG), utilizando-se de um adipômetro calibrado, da marca Lange ${ }^{\circledR}$ (Cambridge Scientific Instruments, Cambridge, MD), de acordo com as técnicas descritas por Lohman e cols. (14). A EDCSe foi tomada no ângulo inferior da escápula obliquamente ao eixo longitudinal, aproximadamente 2 $\mathrm{cm}$ da borda inferior da escápula, seguindo a orientação dos arcos costais. A EDCTri foi determinada paralela- mente ao eixo longitudinal do braço, no ponto médio entre a borda lateral do acrômio e o olécrano. Todas as medidas foram realizadas na posição ortostática no hemicorpo direito e os pontos anatômicos, identificados e marcados antes de serem medidos. Foram pinçados com os dedos polegar e indicador da mão esquerda a uma distância de cerca de $8 \mathrm{~cm}$, em uma linha perpendicular ao eixo da dobra, afastando o tecido muscular subjacente, destacando a dobra a cerca de $1 \mathrm{~cm}$ acima do ponto a ser medido. A dobra foi mantida pressionada enquanto a medida foi realizada e aguardaram-se quatro segundos após a soltura da pressão das hastes para que a leitura do resultado fosse feita.

Todas as medidas foram feitas três vezes, sendo registrado o valor médio. A partir desses dados, foram calculados os percentuais de massa gorda (\%MG EDC), de acordo com as equações propostas por Slaughter e cols. (17) apresentadas abaixo:

Equação l: $\Sigma$ EDC $>35 \mathrm{~mm}: \% \mathrm{MGEDC}=0,546$ $(\Sigma \mathrm{EDC})+9,7$

Equação 2: $\Sigma$ EDC $<35 \mathrm{~mm}: \% \mathrm{MGEDC}=1,33$ $(\Sigma \mathrm{EDC})-0,013(\Sigma \mathrm{EDC})^{2}+2,5$

$\Sigma \mathrm{EDC}=$ somatória da espessura da dobra cutânea tricipital e subescapular.

A partir desses resultados, foram calculados o \%MM (\%MM EDC = $100-\%$ MG EDC) e os valores absolutos de MG [MG EDC $(\mathrm{kg})=$ Peso $\mathrm{x}(\% \mathrm{MG}$ EDC/100)] e MM [MM EDC $(\mathrm{kg})=$ Peso $\mathrm{x}(\% \mathrm{MM}$ EDC/100)].

Após a avaliação dos valores absolutos $(\mathrm{kg})$ de $\mathrm{MG}$ e MM pela BIA e pela EDC, determinaram-se os índices de massa gorda (IMG) e magra (IMM) $\left(\mathrm{kg} / \mathrm{m}^{2}\right)$, dividindo-se a MG ou MM obtida em $\mathrm{kg}$ pelos dois métodos, pelo quadrado da estatura.

Para o arquivo de dados e a análise estatística, foi utilizado o software SPSS for Windows versão 7.5 (SPSS Inc, Chicago). Os dados foram inicialmente tratados por meio de procedimentos descritivos, com cálculos de medianas e valores mínimos e máximos. Foram utilizados os testes não paramétricos de Mann-Whitney para as comparações entre os grupos de casos subdivididos em BC e MC e o grupo total de casos em relação aos controles; o teste de Kruskal-Wallis para a comparação entre o grupo controle e os dois subgrupos dos casos (BC e MC), e o teste de comparações múltiplas para análise de variância não paramétrica para a localização das diferenças quando observadas, além do teste de Wilcoxon para as comparações entre os valores estimados pelos dois métodos (BIA e EDC), e da correlação de Sperman. Todos os resultados foram considerados estatisticamente significativos para $\mathrm{p}<0,05$. 


\section{RESULTADOS}

Na caracterização geral dos grupos casos e controles, não foram observadas diferenças significativas, com exceção do zIMC, que foi significativamente maior no grupo casos. No grupo casos, as pacientes com MC apresentaram valores significativamente maiores de idade cronológica, peso, IMC e quadril em relação às pacientes com BC. Na comparação entre os dois subgrupos de casos (BC e MC) e os controles, apenas o IMC e o quadril foram significativamente diferentes, sendo maiores no grupo MC (Tabela 1 ).

$\mathrm{Na}$ tabela 2, são apresentados os valores relativos (\%) e absolutos (kg) de MG, MM e os valores de IMG e IMM estimados pela BIA e EDC. Na avaliação dos métodos (BIA e EDC) entre si em cada grupo (casos ou controles), não foi observada nenhuma diferença estatística significativa. No entanto, na avaliação entre os grupos (casos e controles), independentemente do método (BIA ou EDC), os valores percentuais, avaliados por BIA, foram significativamente maiores na MG e menores na MM no grupo casos em relação ao grupo controles. O IMG avaliado por BIA e o IMM avaliado por EDC também foram significativamente maiores no grupo casos em relação ao grupo controles. $\mathrm{Na}$ com- paração entre os dois subgrupos casos (BC e MC), os valores absolutos de MM BIA e MM EDC, e IMM BIA e IMM EDC foram significativamente maiores nas pacientes com MC. Quando comparados os dois subgrupos de casos (BC e MC) e os controles, os resultados foram semelhantes aos encontrados na avaliação apenas entre os dois subgrupos de casos.

Quando verificada a correlação entre os valores relativos e absolutos de MM e MG e de IMG e IMM, estimados pelos dois métodos, os valores foram positivos, significativos e elevados em todos os parâmetros analisados (Tabela 3 ).

\section{DISCUSSÃO}

Nessa amostra de mulheres com HAC-D21OH e mulheres saudáveis, os métodos (BIA e EDC) entre si não apresentaram diferenças significativas nos grupos avaliados. Na comparação entre os dois subgrupos de casos (BC e MC), a idade cronológica, o peso, o IMC, o quadril, o MM (BIA e EDC) e o IMM (BIA e EDC) foram maiores no subgrupo MC. O grupo total de casos apresentou valores maiores de zIMC, MG BIA, IMG BIA e IMM EDC, e menores de MM BIA em relação aos controles.

Tabela 1. Valores de mediana (Md), variação (mínimo-máximo) e significância (p) das características gerais de mulheres com hiperplasia adrenal congênita por deficiência clássica da 21-hidroxilase e mulheres saudáveis

\begin{tabular}{|c|c|c|c|c|c|c|c|c|c|c|c|}
\hline & \multicolumn{7}{|c|}{ Casos } & \multicolumn{4}{|c|}{ Controles } \\
\hline & \multicolumn{2}{|c|}{$B C n=10$} & \multicolumn{2}{|c|}{$M C n=6$} & \multirow{2}{*}{$\frac{\mathbf{M}}{\mathbf{p}}$} & \multicolumn{2}{|c|}{ Total $n=16$} & \multicolumn{2}{|c|}{$n=26$} & \multirow{2}{*}{$\frac{M}{p}$} & \multirow{2}{*}{$\frac{K}{p}$} \\
\hline & Md & Variação & Md & Variação & & Md & Variação & Md & Variação & & \\
\hline Idade (anos) & 12,0 & $8,0-16,0$ & 15,5 & $13,0-18,0$ & ${ }^{\star} 0,02$ & 13,0 & $8,0-18,0$ & 13,0 & $8,0-18,0$ & 0,83 & 0,06 \\
\hline 10 (anos) & 11,5 & $7,0-18,0$ & 15,5 & $12,0-18,0$ & 0,89 & 13,5 & $7,0-18,0$ & - & - & - & \\
\hline Peso (kg) & 40,9 & $19,7-93,3$ & 53,0 & $45,9-83,0$ & ${ }^{\star} 0,04$ & 46,5 & $19,7-93,3$ & 45,8 & $23,6-73,2$ & 0,70 & 0,06 \\
\hline zPeso (DP) & $-0,1$ & $-1,8-2,3$ & 0,3 & $-0,4-1,8$ & 0,42 & 0,1 & $-1,8-2,3$ & $-0,3$ & $-1,6-1,4$ & 0,10 & 0,18 \\
\hline Estat (cm) & 143,0 & $112,5-159,0$ & 153,2 & $148,4-163,2$ & 0,07 & 150,5 & $112,5-163,2$ & 155,2 & $125,0-170,3$ & 0,30 & 0,15 \\
\hline zEstat (DP) & $-0,51$ & $-2,72-0,86$ & $-0,6$ & $-2,2-0,1$ & 0,59 & $-0,5$ & $-2,7-0,93$ & $-0,5$ & $-1,5-1,2$ & 0,36 & 0,57 \\
\hline IMC (kg/m²) & 18,3 & $15,5-38,8$ & 22,4 & $19,8-31,5$ & ${ }^{\star} 0,04$ & 20,1 & $15,5-38,8$ & 18,7 & $14,4-25,2$ & 0,19 & ${ }^{\star} 0,02$ \\
\hline zIMC (DP) & 0,5 & $-0,7-2,5$ & 0,6 & $0,4-1,9$ & 0,42 & 0,6 & $-0,7-2,5$ & $-0,1$ & $-1,9-1,1$ & ${ }^{\star} 0,03$ & 0,07 \\
\hline Cintura (cm) & 66,8 & $50,8-109,7$ & 75,7 & $71,0-97,3$ & 0,05 & 71,5 & $50,8-109,7$ & 69,5 & $52,0-78,0$ & 0,34 & 0,06 \\
\hline Quadril (cm) & 81,8 & $63,8-113,0$ & 94,1 & $89,0-113,5$ & ${ }^{\star} 0,02$ & 89,5 & $63,8-113,5$ & 83,0 & $63,0-102,0$ & 0,14 & *0,01 \\
\hline $\mathrm{RCQ}(\mathrm{cm})$ & 0,8 & $0,7-1,0$ & 0,8 & $0,8-0,9$ & 0,91 & 0,81 & $0,73-0,97$ & 0,84 & $0,72-0,99$ & 0,52 & 0,80 \\
\hline EDCTri (mm) & 11,0 & $8,0-33,0$ & 12,5 & $8,0-27,0$ & 0,59 & 22,0 & $9,0-44,0$ & 18,0 & $9,0-26,0$ & 0,14 & 0,29 \\
\hline EDCSe (mm) & 20,0 & $10,0-44,0$ & 24,5 & $9,0-32,0$ & 0,74 & 11,0 & $8,0-33,0$ & 12,5 & $6,0-26,0$ & 0,90 & 0,86 \\
\hline$\Sigma \mathrm{EDC}(\mathrm{mm})$ & 33,5 & $18,0-77,0$ & 38,0 & $18,0-59,0$ & 0,66 & 34,5 & $18,0-77,0$ & 31,5 & $17,0-49,0$ & 0,36 & 0,60 \\
\hline
\end{tabular}

BC: bom controle; MC: mau controle; I0: idade óssea; Estat: estatura; IMC: índice de massa corporal; RCQ: relação cintura/quadril; EDC: espessura de dobras cutâneas; Tri: tricipital; Se: subescapular; $\Sigma$ EDC: somatória da EDCTri + EDCSe; M: teste não paramétrico de Mann-Whitney; K: teste não paramétrico de Kruskal-Wallis; ${ }^{*} \mathrm{p}<0,05$. 
Tabela 2. Valores de mediana (Md), variação (mínimo-máximo) e significância (p) das variáveis de composição corporal de mulheres com hiperplasia adrenal congênita por deficiência clássica da 21-hidroxilase e mulheres saudáveis

\begin{tabular}{|c|c|c|c|c|c|c|c|c|c|c|c|}
\hline & \multicolumn{7}{|c|}{ Casos } & \multicolumn{4}{|c|}{ Controles } \\
\hline & \multicolumn{2}{|c|}{$B C n=10$} & \multicolumn{2}{|c|}{$M C n=6$} & \multirow{2}{*}{$\begin{array}{c}\mathbf{M} \\
\mathbf{p}\end{array}$} & \multicolumn{2}{|c|}{ Total $n=16$} & \multicolumn{2}{|c|}{$\mathbf{n}=\mathbf{2 6}$} & \multirow{2}{*}{$\begin{array}{c}\mathbf{M} \\
\mathbf{p}\end{array}$} & \multirow{2}{*}{$\begin{array}{l}\mathbf{K} \\
\mathbf{p}\end{array}$} \\
\hline & Md & Variação & Md & Variação & & Md & Variação & Md & Variação & & \\
\hline MG BIA (\%) & 28,5 & $17,0-48,0$ & 28,5 & $25,0-44,0$ & 0,87 & 28,7 & $16,7-48,4$ & 24,0 & $13,0-34,0$ & ${ }^{\star} 0,01$ & 0,06 \\
\hline MG EDC (\%) & 27,4 & $17,2-51,7$ & 30,2 & $17,2-41,9$ & 0,66 & 27,9 & $17,2-51,7$ & 26,5 & $16,4-36,5$ & 0,36 & 0,60 \\
\hline MG BIA (kg) & 11,9 & $3,3-45,2$ & 15,1 & $11,5-36,4$ & 0,09 & 13,1 & $3,3-45,2$ & 10,6 & $3,4-18,4$ & 0,09 & 0,06 \\
\hline MG EDC (kg) & 9,9 & $3,4-48,3$ & 15,9 & 8,3-34,8 & 0,13 & 12,0 & $3,4-48,3$ & 11,6 & $3,9-20,3$ & 0,68 & 0,22 \\
\hline MM BIA (\%) & 71,5 & $52,0-83,0$ & 71,5 & $56,0-75,0$ & 0,87 & 71,3 & $51,6-83,3$ & 76,0 & $66-87$ & ${ }^{\star} 0,01$ & 0,06 \\
\hline MM EDC (\%) & 72,6 & $48,3-82,8$ & 69,8 & $58,1-82,8$ & 0,66 & 72,1 & $48,3-82,8$ & 73,5 & $63,5-83,6$ & 0,36 & 0,60 \\
\hline MM BIA (kg) & 29,0 & $16,4-48,1$ & 38,0 & $34,4-46,6$ & ${ }^{\star} 0,02$ & 34,1 & $16,4-48,1$ & 32,6 & $14,6-57,3$ & 0,87 & 0,06 \\
\hline MM EDC (kg) & 30,2 & $16,3-45,0$ & 39,9 & $34,0-48,2$ & ${ }^{\star} 0,02$ & 34,3 & $16,3-48,2$ & 32,7 & $19,7-52,9$ & 0,52 & ${ }^{\star} 0,02$ \\
\hline IMG BIA $\left(\mathrm{kg} / \mathrm{m}^{2}\right)$ & 5,4 & $2,6-18,8$ & 6,3 & $5,1-13,7$ & 0,23 & 5,6 & $2,6-18,8$ & 4,7 & $1,9-7,3$ & ${ }^{\star} 0,02$ & ${ }^{\star} 0,03$ \\
\hline IMG EDC $\left(\mathrm{kg} / \mathrm{m}^{2}\right)$ & 5,2 & $2,7-20,1$ & 6,8 & $3,4-13,1$ & 0,33 & 5,4 & $2,7-20,1$ & 4,9 & $2,4-7,7$ & 0,34 & 0,36 \\
\hline IMM BIA (kg/m²) & 13,2 & $12,2-20,1$ & 16,1 & $14,5-17,9$ & ${ }^{\star} 0,02$ & 14,5 & $12,2-20,0$ & 14,3 & $12,4-19,8$ & 0,52 & ${ }^{\star} 0,02$ \\
\hline IMM EDC (kg/m²) & 14,3 & $12,3-18,8$ & 16,3 & $15,5-18,1$ & ${ }^{\star} 0,01$ & 15,2 & $12,3-18,8$ & 14,3 & $12,0-18,3$ & ${ }^{\star} 0,04$ & ${ }^{*} 0,01$ \\
\hline
\end{tabular}

BC: bom controle; MC: mau controle; MG: massa gorda; MM: massa magra; BIA: impedância bioelétrica; EDC: espessuras de dobras cutâneas; IMG: índice massa gorda; IMM: índice de massa magra; M: teste não paramétrico de Mann-Whitney; K: teste não paramétrico de Kruskal-Wallis; * $p<0,05$.

Tabela 3. Valores de correlação (r) e de significância (p) dos parâmetros da composição corporal estimados pela impedância bioelétrica e pelas espessuras de dobras cutâneas em mulheres com hiperplasia adrenal congênita por deficiência clássica da 21-hidroxilase e em mulheres saudáveis

\begin{tabular}{lcccccc}
\hline & \multicolumn{2}{c}{ Casos $\mathbf{n}=\mathbf{1 6}$} & & \multicolumn{2}{c}{ Controles $\mathbf{~}=\mathbf{2 6}$} \\
\cline { 2 - 3 } \cline { 6 - 7 } & $\mathbf{r}$ & $\mathbf{p}$ & & $\mathbf{r}$ & $\mathbf{p}$ \\
\hline Massa gorda $(\%)$ & 0,62 & 0,01 & & 0,61 & 0,001 \\
Massa gorda $(\mathrm{kg})$ & 0,91 & 0,001 & & 0,89 & 0,001 \\
Massa magra $(\%)$ & 0,62 & 0,01 & & 0,61 & 0,001 \\
Massa magra $(\mathrm{kg})$ & 0,95 & 0,001 & & 0,92 & 0,001 \\
IMG $\left(\mathrm{kg} / \mathrm{m}^{2}\right)$ & 0,85 & 0,001 & & 0,81 & 0,001 \\
IMM $\left(\mathrm{kg} / \mathrm{m}^{2}\right)$ & 0,83 & 0,001 & & 0,74 & 0,001 \\
\hline
\end{tabular}

Teste de Spearman; IMG: índice massa gorda; IMM: índice massa magra.

Em resumo, o grupo casos de forma geral apresentou valores maiores de índices relacionados à gordura corporal (zIMC, MG BIA, IMG BIA) em relação aos controles, porém, quando comparados os casos entre si (BC x MC) e estes em relação aos controles, a amostra apresentou valores maiores de índices de massa magra (MM EDC, IMM BIA e IMM EDC) no grupo casos, especialmente nos MC. Tendo em vista que o grupo com MC apresentou idade cronológica e peso maiores que o BC, é possível que os dados de IMM (BIA e EDC) representem melhor as alterações encontradas que os dados de MM (BIA e EDC).

Isguven e cols. (6) também observaram \%MG avaliado por BIA significativamente maior em um grupo de crianças de ambos os gêneros com HAC em comparação a um grupo controle. Da mesma forma, quando os grupos foram separados por gênero, o \%MG foi significativamente maior nas meninas com HAC que nas meninas do grupo controle, corroborando os resultados do presente estudo.

Estudos demonstram maior risco de sobrepeso e obesidade em crianças e adultos com HAC (4,18-22). Neste estudo, não foram encontradas diferenças no peso corporal e em relação ao IMC; apesar de o grupo de mulheres com HAC-C-D21OH ter apresentado valores maiores em desvio-padrão, os resultados encontrados nos dois grupos casos e controles ficaram dentro dos valores normais de $-2 \mathrm{a}+2$ desvios-padrão. Todavia, $o$ peso e o IMC não são parâmetros suficientemente seguros para a detecção da gordura corporal, já que outros componentes, como músculo e osso, contribuem para seu aumento (23), especialmente em indivíduos com HAC, nos quais o excesso de andrógeno ou o excesso de glicocorticoide podem causar alterações significativas nos componentes de MM e MG (6,21). Por isso, este estudo optou por avaliar a gordura corporal também por EDC e pela BIA.

Uma das hipóteses do aumento do IMC na HAC estaria relacionada ao acréscimo da gordura corporal em decorrência do tratamento com glicocorticoide, que, apesar da busca de um quadro ideal da terapia, utilizando critérios hormonais relativamente sensíveis (androstenediona, 17OH-progesterona, testosterona, 
atividade de renina plasmática), a tentativa de substituir suficientemente a deficiência do glicocorticoide (e/ou mineralocorticoide), com as menores doses possíveis e a distribuição mais satisfatória, não é sempre realizável (6), e a outra hipótese seria a possibilidade do aumento da MM como um possível resultado de excesso de andrógenos $(20,24)$. No entanto, esta última não tem sido confirmada pelos pesquisadores, que demonstram valores mais elevados no \%MG em pacientes com HAC quando comparados com os pares normais, sem encontrarem diferenças significativas em relação à $\mathrm{MM}$ $(20,21,25)$. Em um estudo mais recente, não foram observadas diferenças significativas entre os pacientes jovens (< 30 anos) em relação ao grupo controle, tanto na MM quanto na MG (26). Em todos esses estudos, foi utilizada absorciometria por dupla emissão de raios $\mathrm{X}$ (DXA), método considerado padrão-ouro para a avaliação da composição corporal.

Além disso, Cornean e cols. (18), investigando 14 meninas e oito meninos com HAC, mostraram que o aumento do IMC não se relacionou com a diminuição do crescimento, mas com a adiposidade, refletida no aumento das EDC do tríceps e subescapular.

No presente estudo, foram incluídas somente mulheres para afastar o efeito da diferença de idade no início de tratamento, pois as meninas portadoras de HAC-C-D21OH apresentam ambiguidade genital ao nascimento, o que torna mais fácil o diagnóstico (1), e, portanto, o tratamento inicia-se nos primeiros meses de vida. A idade óssea no grupo casos não apresentou avanço significativo. Além disso, a estatura tanto em valores absolutos como em desvios-padrão foi semelhante entre pacientes e controles, o que, além dos critérios adotados para avaliação do controle da doença, parece demonstrar um bom controle dessa amostra de pacientes. A maturação esquelética não foi realizada no grupo controles por motivos éticos e o pareamento entre o grupo casos e controles foi realizado pela idade cronológica dos controles em relação à idade óssea dos casos, não utilizando o estadiamento puberal devido ao fato de as pacientes do grupo casos poderem apresentar pilificação pubiana acentuada decorrente de hiperandrogenismo atual ou em alguma etapa da vida.

Foram medidos também os perímetros da cintura e do quadril, utilizados para analisar o padrão de distribuição da gordura corporal, além da EDCTri e da EDCSe. Não foram encontrados valores significativamente diferentes nos perímetros da cintura e do quadril, na relação entre a cintura e o quadril, e nas EDC-
Tri e EDCSe na comparação do grupo de casos com os controles. Esses dados diferem em parte do estudo de Isguven e cols. (6), que analisou um grupo de pacientes com HAC de ambos os gêneros e idades entre 1,6 e 10,5 anos. Entretanto, quando esses autores compararam as meninas e os meninos separadamente, as meninas com HAC mantiveram as EDC significativamente maiores nas cinco EDC (bíceps, tríceps, subescapular, suprailíaca e da coxa) e na somatória das cinco EDC, porém as diferenças dos perímetros da cintura e do quadril e RCQ não foram significativas. Na presente amostra, a avaliação comparada entre os casos com BC e MC mostrou que o peso, o IMC e o quadril foram maiores no grupo com $\mathrm{MC}$, o que provavelmente deve estar associado à idade cronológica maior do grupo com MC e esteja também interferindo nos valores de MM e MG (BIA e EDC). Por isso, acredita-se que as alterações encontradas no IMM reflitam melhor as diferenças entre os grupos $\mathrm{BC}$ e MC que os achados de MM (BIA e EDC).

A maior parte dos estudos que investigaram a composição corporal em indivíduos com HAC utilizou o DXA para a avaliação $(20,21,24,26)$. Entretanto, apesar da qualidade dos resultados, esse equipamento, na sua grande maioria, é restrito a poucos ambientes clínicos em razão do seu alto custo operacional (27), o que não ocorre com a BIA e a EDC, que são de menor custo, porém, especialmente em relação à BIA, exigem treinamento adequado do avaliador.

Um fator limitante do presente estudo está no fato de os métodos utilizados (EDC e BIA), apesar das muitas vantagens citadas, serem métodos duplamente indiretos e ainda deixarem dúvidas sobre sua precisão, principalmente pela carência de estudos com portadores de $\mathrm{HAC}-\mathrm{C}-\mathrm{D} 2 \mathrm{lOH}$, nos quais esses métodos não foram testados, utilizando um método padrão-ouro como referência. Embora haja essa limitação, estudos comparando esses métodos, nessa população específica, são inéditos no Brasil e necessários para o aumento das informações e a melhoria da avaliação desses componentes tão importantes para o monitoramento desses pacientes no ambiente clínico e que não possuem aparelhos mais precisos como o DXA.

Segal e cols. (28), comparando os valores obtidos por pesagem hidrostática e BIA em 1.567 indivíduos com grande variação de idade e gordura corporal, confirmaram a validade da técnica de BIA. Entretanto, estudos realizados por Eliakim e cols. (29) e Stewart e Hannan (30) demonstraram que a técnica de EDC 
parece oferecer informações mais precisas sobre a composição corporal que a BIA, quando o DXA é utilizado como método de referência. Vale ressaltar que, no presente estudo, não foram encontradas diferenças significativas entre os valores estimados pela BIA e EDC em nenhuma das variáveis analisadas.

Quando os valores de MG foram ajustados pela estatura (IMG), os resultados foram semelhantes aos da MG (\% e kg), nos quais as diferenças foram observadas apenas nos valores estimados pela BIA. Da mesma forma, outro estudo brasileiro com 21 crianças com HAC$\mathrm{C}-\mathrm{D} 21 \mathrm{OH}$ com a variante PS mostrou valores maiores de $M G$, tanto em valores absolutos quanto em relação à altura, também estimados pela BIA em relação a um grupo controle (25). Em contrapartida, o IMM apresentou valores maiores para os casos quando estimado pelas EDC, sendo o mesmo não observado nos valores de MM estimados por esse mesmo método.

No presente estudo, os resultados fornecidos pela BIA e pela EDC foram semelhantes nos dois grupos, entretanto controversos quando comparados entre estes. A característica singular da amostra demonstra, portanto, que esses métodos devem ser utilizados com cautela. Sugere-se que estudos futuros verifiquem a validade desses instrumentos em pacientes com HAC-C$\mathrm{D} 21 \mathrm{OH}$, utilizando em comparação a métodos de referência, com acurácia comprovada (ex.: DXA), já que a BIA e a EDC foram desenvolvidas a partir de amostra de sujeitos saudáveis.

Agradecimentos: os autores agradecem a Fundação de Amparo à Pesquisa do Estado de São Paulo (Fapesp), ao Conselho Nacional de Desenvolvimento Científico e Tecnológico $(\mathrm{CNPq})$ e à Coordenação de Aperfeiçoamento de Pessoal de Nível Superior (Capes) pelo apoio financeiro concedido.

Declaração: os autores declaram não haver conflitos de interesse científico neste estudo.

\section{REFERÊNCIAS}

1. Merke DP, Bornstein SR. Congenital adrenal hyperplasia. Lancet. 2005;365(9477):2125-36.

2. Riepe FG, Sippell WG. Recent advances in diagnosis, treatment, and outcome of congenital adrenal hyperplasia due to 21-hydroxylase deficiency. Rev Endocr Metab Disord. 2007;8(4):349-63.

3. Torresani T, Biason-Lauber A. Congenital adrenal hyperplasia: diagnostic advances. J Inherit Metab Dis. 2007;30(4):563-75.

4. Jääskeläinen J, Voutilainen R. Growth of patients with 21-hydroxylase deficiency: an analysis of the factors influencing adult height. Pediatr Res. 1997;41(1):30-3.

5. Bonfig W, Bechtold S, Schmidt H, Knorr D, Schwarz HP. Reduced final height outcome in congenital adrenal hyperplasia under prednisone treatment: deceleration of growth velocity during puberty. J Clin Endocrinol Metab. 2007;92(5):1635-9.

6. Isguven P, Arslanoglu I, Mesutoglu N, Yildiz M, Erguven M. Bioelectrical impedance analysis of body fatness in childhood congenital adrenal hyperplasia and its metabolic correlates. Eur $\mathrm{J}$ Pediatr. 2008;167(11):263-8.

7. Lukaski HC, Johnson PE, Bolonchuk WW, Lykken G. Assessment of fat-free mass using bioelectrical impedance measurements of the human body. Am J Clin Nutr. 1985;41(4):810-7.

8. Heyward VH, Stolarczyk LS. Applied body composition assesment. Champaign Illinois: Human Kinetics; 1996.

9. Araújo M, Sanches MR, Suzuki LA, Guerra-Junior G, Farah SB, Mello MP. Molecular analysis of CYP21 and C4 genes in Brazilian families with the classical form of steroid 21-hydroxylase deficiency. Braz J Med Biol Res. 1996;29(1):1-13.

10. Paulino LC, Araujo M, Guerra-Junior G, Lemos-Marini SHV, Mello MP. Mutation distribution and CYP21/C4 locus variability in Brazilian families with the classical form of the 21-hydroxylase deficiency. Acta Paediatr. 1999;88(3):275-83.

11. Lau IF, Soardi FC, Lemos-Marini SHV, Guerra-Junior G, Baptista MTM, Mello MP. H28+C insertion in the CYP21 gene: a novel frameshift mutation in a Brazilian patient with the classical form of 21-hydroxylase deficiency. J Clin Endocrinol Metab. 2001;86(12): 5877-80.

12. Soardi FC, Barbaro M, Lau IF, Lemos-Marini SH, Baptista MT, Guerra-Junior G, et al. Inhibition of CYP21A2 enzyme activity caused by novel missense mutations identified in Brazilian and Scandinavian patients. J Clin Endocrinol Metab. 2008;93(6):2416-20.

13. Tanner JM, Whitehouse RH, Cameron N, Marshall WA, Healy MJR, Goldstein H. Assessment of skeletal maturity and prediction of adult height (TW2 Method). 2.ed. London: Academic Press; 1983.

14. Lohman TG, Roche AF, Martorell R. Anthropometric standardization reference manual. Champaign Illinois: Human Kinetics; 1988.

15. Kuczmarski RJ, Ogden $C L$, Grummer-Strawn LM, Flegal KM, Mei Z, Guo S, et al. CDC growth charts: United States. Adv Data. 2000;8(314):1-27.

16. Sardinha LB, LohmanTG, Teixeira PJ, Guedes DP, Going AB. Comparison of air displacement plethysmography with dual-energy $X$ ray absorptiometry and 3 field methods for estimating body composition in middle-aged men. Am J Clin Nutr. 1998;68(4):786-93.

17. Slaughter MH, Lohman TG, Boileau RA, Horswill CA, Stillman RJ, Van Loan MD, et al. Skinfold equations for estimation of body fatness in children and youth. Hum Biol. 1988;60(5):709-23.

18. Cornean RE, Hindmarsh PC, Brook CG. Obesity in 21- hydroxylase deficient patients. Arch Dis Child. 1998;78(3):261-3.

19. Yu ACM, Grant DB. Adult height in woman with early-treated congenital adrenal hyperplasia (21-hydroxylase type): relation to body mass index in earlier childhood. Acta Paediatr. 1995;84:899-903.

20. Hagenfeldt K, Ritzen EM, Ringertz H, Helleday J, Carlstrom K. Bone mass and body composition of adult women with congenital virilizing 21-hydroxylase deficiency after glucocorticoid treatment since infancy. Eur J Endocrinol. 2000;143(5):667-71.

21. Stikkelbroeck NM, Oyen WJ, Van der Wilt GJ, Hermus AR, Otten BJ. Normal bone mineral density and lean body mass, but increased fat mass, in young adult patients with congenital adrenal hyperplasia. J Clin Endocrinol Metab. 2003;88(3):1036-42.

22. VölkITM, Simm D, Beier C, Dorr HG. Obesity among children and adolescents with classic congenital adrenal hyperplasia due to 21-hydroxylase deficiency. Pediatrics. 2006;117(1):98-105.

23. Guedes DP, Guedes JERP. Manual prático para avaliação em Educação Física. Barueri: Editora Manole; 2006.

24. Cameron FJ, Kaymakci B, Byrt EA, Eberling PR, Warne GL, Wark JD. Bone mineral density and body composition in congenital adrenal hyperplasia. J Clin Endocrinol Metab. 1995;80(7):2238-43. 
25. Santos CTM. Hiperplasia congênita das supra-renais por deficiência da 21-hidroxilase: crescimento e composição corporal de crianças com a forma clássica, variante perdedora de sal. Dissertação de Mestrado - Mestrado em Saúde da Criança e do Adolescente: Universidade Estadual de Campinas; 2007.

26. Falhammar $H$, Filipsson $H$, Holmdahl $G$, Janson PO, Nordenskjold A, Hagenfeldt $K$, et al. Metabolic profile and body composition in adult women with congenital adrenal hyperplasia due to 21-hydroxylase deficiency. J Clin Endocrinol Metab. 2007;92(1):110-6.

27. AniteliTM, Florindo AA, Pereira RMR, Martini LA. Desenvolvimento de equação para estimativa da gordura corporal de mulheres idosas com osteoporose e osteopenia através da espessura de dobras cutâneas tendo como referência absorciometria por dupla emissão de raios X. Rev Bras Med Esporte. 2006;12(6):366-70.

28. Segal KR, Van Loan M, Fitzgrald PI, Hodgdon JA, Van Itallie TB. Lean body mass estimation by electrical impedance analysis: a four-site cross-validation study. Am J Clin Nutr. 1988;47(1):7-14.

29. Eliakim A, Ish-Shalom S, Giladi A, Falk B, Constantini N. Assessment of body composition in ballet dancers: correlation among anthropometric measurements, bio-electrical impedance analysis, and dual-energy X-ray absorptiometry. Int J Sports Med. 2000;21(8):598-601.

30. Stewart AD, Hannan WJ. Prediction of fat and fat-free mass in male athletes using dual X-ray absorptiometry as the reference method. J Sports Sci. 2000;18(4):263-74. 